\title{
Análisis del impacto del Covid - 19 en las ventas de una empresa de autopartes de la provincia de Jaén, Perú
}

\author{
Mg. CPC. Víctor Hugo Puican Rodríguez \\ https://orcid.org/0000-0001-7402-9576 \\ victor-puican@hotmail.com \\ Universidad Nacional de Trujillo \\ Trujillo - Perú
}

\section{RESUMEN}

El trabajo titulado: Análisis del impacto del COVID 19 en las ventas de una empresa de autopartes de la provincia de Jaén, tuvo como objetivo general determinar el impacto del COVID-19 en las ventas de una empresa de autopartes de la provincia de Jaén. A través de un estudio descriptivo, con análisis documental y observación, permitieron obtener como resultado, que el COVID - 19 que la disminución de las ventas de una empresa de autopartes de la provincia de Jaén, si se debió por el aislamiento social que decretó el gobierno con el propósito de paralizar el contagio por el COVID - 19, siendo el mes con más caída julio del 2020, con el 99\%, estos datos permitieron concluir que, dicho impacto, es muy significativo, afectando directamente los estados financieros de esta organización, lo cual no han podido cumplir con sus pasivos corrientes y no corrientes programados en casi 09 meses, ocasionándole problemas laborales, como judiciales por este incumplimiento, de la misma manera las medidas tomadas por el gobierno no han podido subsanar estos problemas en su totalidad, debiendo la empresa acceder al reactiva Perú con el propósito de cubrir algunas obligaciones económicas que ha generado el cierre de las empresas por el tema del aislamiento social.

Palabras clave: Registro de ventas e ingresos, estados financieros, aislamiento social. 


\title{
Analysis of the impact of Covid - 19 on the sales of an auto parts company in the province of Jaen, Peru.
}

\begin{abstract}
The general objective of the study entitled: Analysis of the impact of COVID-19 on the sales of an auto parts company in the province of Jaén, was to determine the impact of COVID-19 on the sales of an auto parts company in the province of Jaén. Through a descriptive study, with documentary analysis and observation, it was possible to obtain as a result, that COVID - 19 that the decrease in sales of an auto parts company in the province of Jaén, if it was due to the social isolation decreed by the government with the purpose of paralyzing the contagion by COVID 19, being the month with more fall July 2020, with $99 \%$, these data allowed to conclude that, such impact, is very significant, directly affecting the financial statements of this organization, which have not been able to meet their current and non-current liabilities scheduled in almost 09 months, causing labor problems, as judicial for this breach, in the same way the measures taken by the government have not been able to remedy these problems in full, the company must access the reactive Peru in order to cover some economic obligations that has generated the closure of companies on the issue of social isolation.
\end{abstract}

Key words: Sales and income records, financial statements, social isolation. 


\section{INTRODUCCIÓN}

Recuerdo que, cuando se escuchó el primer caso en China nadie en el mundo creía que esto se expandió tan rápido a nivel mundial, porque se pensó que esto sería controlado en el mismo país oriental, cometiendo el grave error de confiarse, es por este y otros factores que las organizaciones han visto afectado sus ventas durante el primer día que se decretó el confinamiento social.

Los ingresos han disminuido en las organizaciones de una manera apresurada, viendo afectado sus estados financieros, trayendo como consecuencia que estas organizaciones no cumplan con sus pasivos corrientes, como sueldos de sus trabajadores, pago de cuotas de créditos, beneficios sociales de sus trabajadores, servicios, etc. Sin embargo, estas situaciones podrían revertirse a medida que la pandemia se desvanezca en el Ecuador, los datos históricos indican que las consecuencias económicas a largo plazo podrían persistir durante al menos una generación, y si a esto le agregamos el prolongado período de tasas de interés reales deprimidas algo parecido a un estancamiento secular que podría durar al menos dos décadas (Vásquez, Quevedo, \& Quevedo, 2020).

El efecto del COVID 19 es a nivel mundial, pero cada país debe enfrentar el problema teniendo en cuenta sus recursos y sus activos intangibles con el fin de enfrentar esta crisis y apoyar a las organizaciones a través de herramientas económicas y fiscales que permitan surgir como el ave fénix de las cenizas y puedan posicionarse nuevamente en el mercado, pero lamentablemente el gobierno colombiano enfrenta una gran inestabilidad sociopolítica, tras las protestas masivas en los últimos meses del 2019, y si a esto le sumamos las malas perspectivas económicas para 2020, el cual viene siendo producido por la bajada en los precios del petróleo y la desaceleración económica regional, y la realidad de una desigualdad estructural, siendo estos factores los que vienen provocando que la pandemia tenga un impacto desproporcionado en algunos sectores sociales (Rodríguez, 2020).

De acuerdo a CEPAL (2020), en este 2020, el impacto del COVID 19 en el PIB mundial se ha reducido un 5,2\%. La caída en los países con economías desarrolladas es el 7,0\% y en los países con economías emergentes el 1,6\% (p.2), al tener esta caída abismal en la economía mundial disminuye el poder adquisitivo de los clientes de todas las organizaciones, generando una crisis financiera dentro de ellas, por el motivo de que sus ventas se ven afectadas, debiendo tomar decisiones de manera urgente con el fin de adaptarse a los cambios no solo de la economía, sino de los hábitos de sus potenciales clientes. 
De acuerdo a Jaramillo y Ñopo (2020), manifiesta que en nuestro país se han tomado medidas para las empresas que han sido orientadas en evitar el corte de la cadena de pagos como el despido masivo de sus trabajadores, contando con un paquete de garantías crediticias por hasta el $98 \%$ de los nuevos créditos que tomen las empresas en el sistema financiero nacional. Pero estas medidas solo favorecen a las empresas que se encuentran formalizadas y que hayan tenido ingresos en el primer trimestre de este año 2020, dependiendo de esto es el préstamo que estas podrían obtener.

En cambio, Canseco (2020). Revela que a pesar que las empresas formales tienen mayor liquidez, canales de amortiguamiento de crisis están sufriendo y algunas están casi quebradas, imagínense el informal, el emolientero, el que vendía en la calle, su capacidad de ahorro se ha destrozado, no tiene ningún capital.

Se puede concluir que, el aislamiento social ha impactado fuertemente a la economía de las empresas, en unas más que otras, siendo en estos puntos buscar las medidas que nos lleven a aprovechar las oportunidades que viene generando la pandemia. Es por este motivo que he decidido realizar esta investigación para poder detectar si la pandemia generada por el COVID -19 tiene el efecto en la disminución de las ventas.

Este trabajo se justifica por la teoría de la partida doble, donde Castillo (2015), mencionar que la partida doble surgió a principios del siglo XIV según consta en algunos registros comerciales aparecidos en pergaminos encontrados en Milán- Italia. Sin embargo, fue Fray Lucas Pacciolo, monje franciscano, quien puntualizó y divulgó los procedimientos contables en base a la Partida Doble, la cual tiene carácter de Universalidad, teniendo como pilares de sustento contable: El Debe y El Haber. Siendo desde ese momento denominado como el padre de la contabilidad hasta la actualidad.

González y Flores (2020). Decidió analizar la cultura organizacional y sustentabilidad empresarial en las pymes durante los períodos de confinamiento social. Fue cuantitativa, de tipo descriptivo, con cuestionario a 14 PYMES. Llegando a concluir que el impacto de la pandemia en la sustentabilidad de la empresa ha generado problemas de liquidez afectando seriamente la estabilidad económica de las PYMES. En cambio, Carbó et al., (2020). Determinó la recuperación Financiera de las Empresas tras el COVID-19. Fue de tipo cualitativo, concluyendo que, dada la incertidumbre económica actual, parece esencial la flexibilidad de los programas de avales, de modo que si se agotan puedan extenderse en importe y en el tiempo. De lo contrario, es probable que sean insuficientes. Las previsiones actuales apuntan a que podrían ser también necesarios extender los programas durante 2021. En todos los casos, financiación orientada a 
resolver problemas financieros aparejados al Covid-19, donde la eficiencia y buen uso de los participantes privados en esta financiación jugarán también un papel determinante. La demanda que están recibiendo las entidades financieras es muy elevada, por lo que ampliar el programa, bajo estos puestos, parece conveniente. Asimismo, Blanco (2020). Decidió analizar el impacto del COVID.19 en la economía latinoamericana. De tipo descriptivo. Concluyendo que en América Latina el impacto será desigual entre países, más profundo en aquellos que mostraban mayores vulnerabilidades antes de la llegada del COVID-19. También será desigual la recuperación, más vigorosa en aquellas economías que partían de una mejor situación.

Pero Paez (2020). Diagnóstico las consecuencias económicas de la pandemia COVID-19. Fue de tipo explicativo, concluyendo que los países deben realizar grandes inversiones en materia de beneficios para estos empleados como para la infraestructura teniendo claro que las cuarentenas decretadas en las naciones no son para acabar el virus sino para poder tener tiempo para preparar el sistema de salud de no tener una sobre ocupación en las unidades de cuidado intensivo porque en caso de llegar a este punto el virus sería mortal y no habría como poder contener y atender la gente contagiada. En cambio, Clavellina (2020). Analizó las implicaciones económicas de la pandemia por COVID-19 y opciones de política. Fue de tipo aplicativo, llegando a concluir que, las economías emergentes muestran un panorama menos alentador. La debilidad institucional y financiera que los caracteriza podría limitar su respuesta ante la crisis, por lo que se requiere de acciones coordinadas en conjunto con las economías avanzadas.

Con respecto a los trabajos previos, se consideró el estudio de Ccaccro y Panéz (2020). Analizó el impacto en la rentabilidad de la NIC 19: "Beneficios de los empleados" en las empresas del sector transporte terrestre de Lima Este en el periodo 2018. Fue de tipo descriptiva, de enfoque cuantitativo, la técnica fue la entrevista. Llegando a concluir que establecer el impacto que genera la aplicación de la Norma Internacional de Contabilidad 19: "Beneficios a los empleados" en las empresas del sector transporte terrestre de Lima Este en el periodo 2018, ya que, si no se refleja de forma correcta en los Estados Financieros, los usuarios podrían tomar decisiones erradas.

Pero, Jaramillo y Ñopo (2020). Decidió observar y analizar el impacto del Covid-19 sobre la economía peruana. Fue de tipo explicativa, se utilizó la técnica de la observación. Llegando a concluir que la magnitud del esfuerzo de aplicar políticas en la segunda etapa va a depender tanto de cuánto se prolongue la cuarentena indiscriminada como de cuán efectivas en mantener a las empresas con vida hayan sido las políticas de la primera etapa. Entonces, es clave que se 
implementen estrategias de evaluación continua de los efectos de las medidas que se han ido tomando, con el fin de mejorar su gestión y efectividad.

Asimismo, Hernández y Zevallos (2020). Determinó estudiar el impacto económico del estado de emergencia por la pandemia de COVID-19 en los trabajadores y trabajadoras de las artes visuales en el Perú. Fue cualitativa, descriptiva, concluyendo que la información confirma los temores de buena parte del sector cultural: hay una sensación generalizada de desconcierto e incertidumbre frente a lo que vendrá. A partir de un análisis de los resultados y de la revisión de propuestas de otras partes del mundo, nos gustaría plantear una serie de medidas para la acción a mediano y largo plazo.

Con respecto a las bases teóricas, las ventas, se afianzan en la teoría de la partida doble, donde Corvellec (2001), manifiesta que la técnica denominada partida doble "ha sido desarrollada originariamente por mercaderes italianos a inicios del siglo XIII" (p.3); esto fue generado por el incremento de las sociedades y relaciones comerciales con otros países, convirtiendo las actividades económicas y transacciones financieras muy complejas. Existiendo estas múltiples necesidades comenzaron a desarrollar un sistema que les permitiera manejar y controlar sus cuentas por cobrar y sus cuentas por pagar, es ahí donde crearon el sistema de registro de transacciones comerciales que actualmente llamamos contabilidad.

A criterio de Castillo (2015), la partida doble surgió a principios del siglo XIV según consta en algunos registros comerciales aparecidos en pergaminos encontrados en Milán- Italia.

Sin embargo, fue Fray Lucas Pacciolo, monje franciscano, quien puntualizó y divulgó los procedimientos contables en base a la Partida Doble, la cual tiene carácter de UNIVERSALIDAD, teniendo como pilares de sustento contable: EL DEBE Y EL HABER. Siendo desde ese momento denominado como el padre de la contabilidad hasta la actualidad.

Las ventas se pueden definir, como la operación mediante la cual una persona transmite a otra persona la propiedad que tiene sobre un bien o derecho, a cambio de un precio determinado (Vásquez G. , 2019)

Asimismo, es la forma de acceso al mercado que es practicada por la mayor parte de las empresas que tiene una saturación en su producción y cuyo objetivo es vender lo que producen, en lugar de producir lo que el mercado desea (Thompson, 2019)

Del mismo modo, es otra forma de acceso al mercado para muchas empresas, cuyo objetivo es vender lo que hacen en lugar de hacer lo que el mercado desea" (Kotler, 2018).

De estas definiciones se puede afirmar que la venta, es el proceso mediante el cual las empresas entregan sus bienes o servicios a un tercero con el fin de recibir por esta prestación un ingreso 
económico el cual deberá cubrir los costos y gastos que se ejecutaron para su producción, comercialización y entrega del producto a ese tercero.

Lo importante de las ventas, es que son esenciales para las empresas ya que les permiten dar salida a sus productos o prestar los servicios que estas ofrecen a sus clientes y potenciales clientes con el fin de obtener ingresos económicos, ya que mediante esos ingresos ellos podrán cumplir con sus pasivos corrientes y no corrientes asumidos durante el desarrollo de cada de una de sus actividades.

De la misma manera las ventas le permiten a la empresa ver qué productos o si sus servicios son rentables con el fin de poder visualizar la viabilidad de su negocio, y todo esto se podrá observar en el balance de comprobación y en los demás estados financieros.

Asimismo, se define al registro de ventas, como un libro auxiliar obligatorio de foliación doble, la legalización se realiza en la primera página ya sea libro o reporte por computadora (SUNAT, 2018). Este libro es importante porque sirve para registrar las operaciones de ventas de mercaderías, bienes o servicios de una empresa (SUNAT, 2018).

De acuerdo a la SUNAT (2018). Indica que el rayado del registro de ventas debe tener como mínimo debe contener los siguientes datos: Primero se debe colocar el periodo que se está trabajando, asignar el RUC de la empresa, asignar los apellidos y nombres, denominación o razón social, número Correlativo del registro o código único de la operación, fecha de emisión del comprobante de pago emitido por el proveedor (factura, boleta, ticket, nota de débito o crédito comercial) (SUNAT, 2018).

De la misma forma debe contener la fecha de vencimiento y/o pago, comprobante de pago o documento: Aquí debes asignar el tipo de comprobante, la serie y el número del mismo, información del cliente: aquí se debe agregar el tipo de documento y su respectivo número, de la misma manera debes asignar los apellidos y nombres o razón social del cliente, la base imponible de la operación gravada, IGV y/o IPM y el importe total del comprobante de pago.

La dimensión considerada para este caso fue el registro de ventas, que es el documento legal tributario donde se agregan todos los ingresos generados por las operaciones económicas que realizan las empresas. Estos ingresos deben ser por la actividad principal que tiene registrada la empresa en la SUNAT (SUNAT, 2018).

Este registro permite a la SUNAT verificar y controlar los ingresos que las empresas declaran a través del Programa de Libros Electrónicos, con el fin de determinar si los impuestos cancelados son los correctos, caso contrario tendrán que cancelar una multa por presentación de documentación falsa (SUNAT, 2018). 
Asimismo, se consideraron algunas definiciones de materia financiera, donde la actividad económica, es un proceso en el cual las personas producen bienes y prestan servicios para cubrir las necesidades de terceras personas a cambio de una retribución. Se clasifican de acuerdo al sector al que pertenecen (Salvador, 2017).

En cambio, el activo, es un depósito de valor que genera un beneficio o una serie de beneficios para su propietario económico al ser poseído o utilizado durante un período. Es un medio que permite transferir valor de un período contable a otro (Salvador, 2017).

El balance es un estado contable, confeccionado en un momento concreto, de los valores de activos poseídos y de los pasivos contraídos por una unidad institucional o un grupo de ellas (Salvador, 2017).

Los bienes, son todos los objetos físicos para los cuales existe una demanda, sobre los cuales pueden establecerse derechos de propiedad, y cuya propiedad puede ser transferida de una unidad institucional a otra, a través de una operación en el mercado (Salvador, 2017).

La cuenta contable, es un instrumento que permite registrar, para un determinado aspecto de la vida económica: a) los empleos y los recursos; o b) las variaciones de activos y las variaciones de pasivos; o c) las existencias de activos y pasivos que existen en un determinado momento (Salvador, 2017).

Las existencias, son activos consistentes en bienes y servicios producidos durante el período actual o en un período anterior, y que son mantenidos para ser vendidos, ser utilizados en la producción o ser destinados a otro uso en una fecha posterior. (Salvador, 2017).

Los ingresos ordinarios, es la entrada bruta de beneficios económicos, durante el periodo, surgidos en el curso de las actividades ordinarias de una entidad (MEF, 2017).

El valor razonable, es el importe por el cual puede ser intercambiado un activo, o cancelado un pasivo, entre un comprador y un vendedor interesados y debidamente informados, que realizan una transacción libre (MEF, 2017).

\section{ESTRATEGIAS METODOLÓGICAS}

En este trabajo se consideró como objetivo general determinar el impacto del COVID-19 en las ventas de una empresa de autopartes de la provincia de Jaén. Asimismo, se utilizó el método empírico de la investigación científica, porque tolera toda una serie de instrucciones prácticas coherentes con el objeto de estudio y los medios de investigación que admiten revelar las peculiaridades esenciales y relaciones principales del mismo, estas particularidades son asequibles a la admiración sensorial. Personifican un nivel en el juicio de indagación cuyo contenido proviene de la práctica, el cual es sometido a cierta transformación legítima. Del 
mismo modo, se utilizó el método de observación científica, porque consiste en la inteligencia dirigida del objeto de la exploración accediendo identificar el contexto mediante la penetración continua de los objetos y anómalos, se esgrimió en diferentes instantes de una investigación, en su etapa inicial se emplea como análisis de la dificultad a estudiar.

La muestra estuvo conformada por los reportes de ventas de enero a diciembre 2019 y 2020 . Las técnicas que se van a utilizar es la observación el cual nos permitió evidenciar los datos de los reportes económicos de la empresa. Asimismo, se manipuló la técnica análisis documental, el cual nos permitió determinar si el COVID-19 ha tenido un impacto en las ventas de una empresa de autopartes de la provincia de Jaén. De la misma manera se utilizaron software de procesamiento de datos como Microsoft Word 2010, Microsoft Excel 2010 y el SPSS vr. 26.

\section{RESULTADOS Y DISCUSIÓN}

Para determinar los resultados se analizaron los reportes de cada registro de ventas de forma mensual de los periodos enero a diciembre del 2019 y 2020.

Primer objetivo específico: Analizar las ventas de enero a diciembre 2019- 2020.

\section{Tabla 1.}

Análisis comparativo de las ventas de enero a diciembre 2019 - 2020

\begin{tabular}{lll}
\hline & Períodos & \\
\cline { 2 - 3 } Mes & $\mathbf{2 0 1 9}$ & $\mathbf{2 0 2 0}$ \\
\hline Enero & 37587.08 & 115875.32 \\
Febrero & 33446.93 & 135875.21 \\
Marzo & 37498.58 & 30258.32 \\
Abril & 76409.89 & 4823.23 \\
Mayo & 33512.93 & 1258.36 \\
Junio & 73288.46 & 857.12 \\
Julio & 73998.47 & 936.12 \\
Agosto & 72832.10 & 9578.25 \\
Septiembre & 75011.79 & 29784.15 \\
Octubre & 38065.75 & 45546.89 \\
Noviembre & 72079.69 & 58798.65 \\
Diciembre & 37123.72 & 82587.12 \\
\hline TOTALES & $\mathbf{1 0 2 0 8 5 5 . 3 9}$ & $\mathbf{5 1 6 1 7 8 . 7 4}$ \\
\hline
\end{tabular}

Fuente: Reportes de registro de ventas 2019 - 2020 de la empresa

De acuerdo a los resultados reflejados en la tabla 2, se evidencia que en comparación de los meses de enero y febrero 2019 con el 2020, hubo un incremento considerable en enero el $32 \%$ y en febrero el 51,50 \% 2020, llegando a obtener un incremento de las ventas en ambos meses de S/ 80716.52 (Ochenta mil setecientos dieciséis con 52/100 soles) proyectando un año productivo y con aras al crecimiento económico de la empresa. En cambio, desde que se inició en el mes de marzo 2020 el aislamiento social por la pandemia del COVID - 19, iniciaron los problemas 
para la empresa, porque comenzó a disminuir el nivel de ventas en comparación con los mismos meses del 2019; en el mes de marzo tuvieron una caída del 34,50\% en comparación con el mismo mes del 2019, en el mes de abril tuvo una caída del 93,50\%, en el mes de mayo tuvo una caída del 98,30\%, en junio el 98,50\%, en julio el 99\%, en agosto el 98\%, en septiembre el 97\%, en octubre el $51,50 \%$, en noviembre el $81,20 \%$ y en diciembre el $8 \%$, llegando a tener una pérdida de S/ 585393.17 (quinientos ochenta y cinco mil trescientos noventa y tres con 17/100 soles), evidenciando de esta manera que el impacto de la pandemia del COVID - 19 en las ventas de esta empresa ha sido muy fuerte, generando problemas de liquidez para afrontar sus pasivos corrientes y no corrientes

Tabla 2

\section{Periodos de baja de las ventas del 2020}

\begin{tabular}{ll}
\hline Mes & $\%$ \\
\hline Marzo & 34.50 \\
Abril & 93.50 \\
Mayo & 98.30 \\
Junio & 98.50 \\
Julio & 99.00 \\
Agosto & 98.00 \\
Septiembre & 97.00 \\
Octubre & 51.50 \\
Noviembre & 31.20 \\
Diciembre & 3.00 \\
\hline
\end{tabular}

Fuente: Reportes de registro de ventas 2019 - 2020 de la empresa.

De acuerdo a los resultados reflejados en la tabla 3, nos revela que la caída de las ventas en una empresa de autopartes de la provincia de Jaén, inician desde el mes de marzo del 2020 de forma acelerada, siendo los meses con mayor caída en sus ventas julio con una reducción del 99\%, seguido por junio con el 98,50\%, continuando con mayo con el 98,30\%, seguido por agosto con el $98 \%$, seguido por septiembre con el $97 \%$, seguido por abril con el 93,50\% y finalmente noviembre con el 81,20\%, demostrando de esta manera que el impacto del COVID-19 fue negativo en las ventas de esta empresa, generando problemas económicos debiendo tomar medidas drásticas en no cumplir con el pago de sus cuotas de los préstamos obtenidos, de la misma forma tuvo que acogerse a la suspensión perfecta de labores de sus trabajadores, con el propósito de reducir los gastos operativos. 


\section{Tabla 3}

Evaluar el impacto del COVID - 19 en las ventas de una empresa de autopartes de la provincia de Jaén

\begin{tabular}{lll}
\hline MES & $\mathbf{2 0 1 9}$ & $\mathbf{2 0 2 0}$ \\
\hline Enero & 37587.08 & 115875.32 \\
Febrero & 33446.93 & 135875.21 \\
Marzo & 37498.58 & 30258.32 \\
Abril & 76409.89 & 4823.23 \\
Mayo & 33512.93 & 1258.36 \\
Junio & 73288.46 & 857.12 \\
Julio & 73998.47 & 936.12 \\
Agosto & 92832.10 & 9578.25 \\
Septiembre & 95011.79 & 29784.15 \\
Octubre & 38065.75 & 45546.89 \\
Noviembre & 72079.69 & 58798.65 \\
Diciembre & 37123.72 & 82587.12 \\
\hline
\end{tabular}

Fuente: Reportes de registro de ventas 2019 - 2020 de la empresa.

De acuerdo a los resultados reflejados en la tabla 4, nos revela que el impacto del COVID - 19 en las ventas en la empresa de autopartes de la provincia de Jaén, es muy significativo, el cual, afectando directamente los estados financieros de esta organización, el cual no han podido cumplir con sus pasivos corrientes y no corrientes programados en casi 09 meses, ocasionándole problemas laborales, como judiciales por este incumplimiento, de la misma manera las medidas tomadas por el gobierno no han podido subsanar estos problemas en su totalidad, debiendo la empresa acceder al reactiva Perú con el propósito de cubrir algunas obligaciones económicas que ha generado el cierre de las empresas por el tema del aislamiento social. 


\section{CONCLUSIÓN O CONSIDERACIONES FINALES}

Al realizar el análisis de las ventas de enero a diciembre 2019- 2020. Se evidenciaron que los problemas para la empresa, iniciaron en el mes de marzo del 2020, porque tuvieron una caída del $34,50 \%$ en comparación con el mismo mes del 2019, en el mes de abril tuvo una caída del 93,50\%, en el mes de mayo tuvo una caída del 98,30\%, en junio el 98,50\%, en julio el 99\%, en agosto el $98 \%$, en septiembre el $97 \%$, en octubre el 51,50\%, en noviembre el $81,20 \%$ y en diciembre el $8 \%$, evidenciándose de esta manera que el impacto de la pandemia del COVID 19 en las ventas de esta empresa ha sido muy fuerte, generando problemas de liquidez para afrontar sus pasivos corrientes y no corrientes

Al proceder con identificar los periodos de baja de las ventas 2020, se observó que la caída de las ventas en la empresa dedicada a la venta de autopartes de la provincia de Jaén, inician desde el mes de marzo del 2020 de forma acelerada, siendo los meses con mayor caída en sus ventas julio con una reducción del 99\%, seguido por junio con el 98,50\%, continuando con mayo con el $98,30 \%$, seguido por agosto con el $98 \%$, seguido por septiembre con el 97\%, seguido por abril con el $93,50 \%$ y finalmente noviembre con el $81,20 \%$, demostrándose de esta manera que el impacto del COVID-19 fue negativo en las ventas de esta empresa, generando problemas económicos debiendo tomar medidas drásticas en no cumplir con el pago de sus cuotas de los préstamos obtenidos, de la misma forma tuvo que acogerse a la suspensión perfecta de labores de sus trabajadores, con el propósito de reducir los gastos operativos.

Al evaluar el impacto del COVID-19 en las ventas de la empresa dedicada a la venta de autopartes de la provincia de Jaén. Se determina que dicho impacto, es muy significativo, el cual, afectando directamente los estados financieros de esta organización, el cual no han podido cumplir con sus pasivos corrientes y no corrientes programados en casi 09 meses, ocasionándole problemas laborales, como judiciales por este incumplimiento, de la misma manera las medidas tomadas por el gobierno no han podido subsanar estos problemas en su totalidad, debiendo la empresa acceder al reactiva Perú con el propósito de cubrir algunas obligaciones económicas que ha generado el cierre de las empresas por el tema del aislamiento social. 


\section{LISTA DE REFERENCIAS}

Blanco, A. (6 de 05 de 2020). El profundo, pero transitorio, impacto del COVID-19 en la economía latinoamericana. Real Instituto ELCANO, 9. Obtenido de http://www.realinstitutoelcano.org/wps/wcm/connect/df97a839-bc5e-4325-a10f7c405596c222/ARI63-2020-Blanco-profundo-pero-transitorio-impacto-COVID-19-eneconomia-latinoamericana.pdf?MOD=AJPERES\&CACHEID=df97a839-bc5e-4325-a10f$7 \mathrm{c} 405596 \mathrm{c} 222$

Canseco, R. (04 de 2020). Economía Peruana: Contención y Reactivación en tiempos de COVID -19. Universidad San Ignacio de Loyola, 36. Obtenido de http://repositorio.usil.edu.pe/bitstream/USIL/9889/1/2020_Diez-Canseco_Econom\%c3\%adaPeruana.pdf

Carbó, S., \& Et al. (06 de 2020). Por la Recuperación Financiera de las Empresas tras el COVID-19. Fedea Policy Papers, 13. Obtenido de http://documentos.fedea.net/pubs/fpp/2020/06/FPP202009.pdf

Castillo, J. (2015). Teoría de la partida doble. En J. Castillo, Teoría de la partida doble (pág. 125). Madrid, Madrid, España.

Ccaccro, J., \& Panéz, K. (2020). NIC 19: Beneficios a los empleados y su impacto en la rentabilidad de las empresas del sector transporte terrestre de Lima Este en el periodo 2018. tesis, Universidad Peruana de Ciencias Aplicadas, Lima, Lima, Perú. doi:http://hdl.handle.net/10757/652517

CEPAL. (2020). Enfrentar los efectos cada vez mayores del COVID-19 para una reactivación con igualdad: nuevas proyecciones. Especial, CEPAL, Buenos Aires, Buenos Aires, Argentina. Obtenido de https://repositorio.cepal.org/bitstream/handle/11362/45782/S2000471_es.pdf?sequence=4\&isA llowed=y

Clavellina, J. (04 de 2020). Implicaciones económicas de la pandemia por COVID-19 y opciones de política. Instituto Belisario Doínguez, $11 . \quad$ Obtenido de http://www.bibliodigitalibd.senado.gob.mx/bitstream/handle/123456789/4829/NE_coronavirus _implicaciones\%20econ\%c3\%b3micas\%20_010422020.pdf?sequence=1\&isAllowed=y

Corvellec, H. (Julio de 2001). La contabilidad por partida doble. Heterogénesis, 10. Obtenido de https://www.redalyc.org/pdf/108/10803602.pdf

Gozález, R., \& Flores, K. (03 de 04 de 2020). Cultura organizacional y Sustentabilidad empresarial en las Pymes durante crisis períodos de confinamiento social. Centro Internacional de 


\section{Investigación}

Desarrollo,

12.

Obtenido

de

https://ciidjournal.com/index.php/abstract/article/view/40/39

Hernández, M., \& Zevallos, C. (03 de 2020). Impacto económico del estado de emergencia por la pandemia de COVID-19 en los trabajadores y trabajadoras de las artes visuales en el Perú. Facultad de Ciencias y Artes de la Comunicación, 16. Obtenido de http://curadoresdelperu.org/wp-content/uploads/2020/04/Impacto-COVID19_Artes-

Visuales_Peru\%CC\%81-2.pdf

Jaramillo, M., \& Ñopo, H. (03 de 2020). COVID -19 y el schock externo: Impactos económicos y opciones de política en el Perú. Análisis para el Desarrollo, 28. Obtenido de https://proactivo.com.pe/wp-content/uploads/2020/04/Covid-19-y-el-schock-externo-Impactosecon\%C3\%B3micos-y-opciones-de-pol\%C3\%ADtica-en-el-Per\%C3\%BA.pdf

Jaramillo, M., \& Ñopo, H. (09 de 2020). El impacto del Covid-19 sobre la economía peruana. Journal of Economic Literature, $11 . \quad$ Obtenido de http://revistaeconomia.unam.mx/index.php/ecu/article/view/552/585

Kerfant. (2019). El margen comercial. En Kerfant, El margen comercial (pág. 32). Bogotá, Bogotá, Colombia. Obtenido de https://blog.cofike.com/el-margen-comercial-como-se-calcula-y-paraque-sirve/

Kotler, P. (2018). Dirección de Mercadotecnia. En P. Kotler, Dirección de Mercadotecnia (Octava Edición ed., pág. 15). Berlín, Berlín, Alemania.

MEF. (2017). NIC 18 Ingresos. En MEF, NIC 18 Ingresos (pág. 18). Bogotá, Bogotá, Colombia. Obtenido de https://www.mef.gob.pe/contenidos/conta_publ/con_nor_co/NIC18_04.pdf

OBS. (2018). Ratios financieros, fórmulas que debes conocer. En OBS, Ratios financieros, fórmulas que debes conocer (pág. 12). Barcelona, Barcelona, España. Obtenido de https://obsbusiness.school/es/blog-investigacion/finanzas/ratios-financieros-formulas-quedebes-conocer

Paez, F. (2020). Consecuencias económicas de la pandemia COVID-19 en América Latina. Creative commons, 14. Obtenido de https://repository.ucatolica.edu.co/bitstream/10983/24648/1/Consecuencias\%20econ\%c3\%b3 micas\%20de\%20la\%20pandemia\%20COVID\%20final.pdf

Rodríguez, É. (30 de 04 de 2020). Impacto económico, social y político de la COVID-19. Análisis Carolina, 14. Obtenido de file://C:/Users/HUGO/Downloads/Dialnet-Colombia-7439286.pdf 
Salvador, B. C. (2017). Glosario de términos técnicos y conceptos económicos. En B. C. Salvador, Glosario de términos técnicos y conceptos económicos (pág. 20). El Salvador, El Salvador, El Salvador. Obtenido de https://www.bcr.gob.sv/bcrsite/uploaded/content/cuadro/776346701.pdf SUNAT. (2018). Registro de compras. En SUNAT, Registro de compras (pág. 15). Lima, Lima, Perú. Thompson, I. (2019). Concepto de venta. En I. Thompson, Concepto de venta (pág. 21). Lima, Lima, Perú.

Vásquez, G. (2019). Las ventas en el contexto gerencial latinoamericano. En G. Vásquez, Las ventas en el contexto gerencial latinoamericano (pág. 7). Bogotá, Bogotá, Colombia. Obtenido de https://ucema.edu.ar/cimei-base/download/research/59_Vasquez.pdf

Vásquez, L., Quevedo, M., \& Quevedo, J. (22 de 07 de 2020). Gerencia de Emprendimientos en Época de Pandemia. Ciencias Económicas y Empresariales, 17. Obtenido de https://fipcaec.com/index.php/fipcaec/article/view/256/430 